$x=\frac{1}{2}\left\{3 d_{j}+\sqrt{4 t_{j}-3 d_{j}^{2}}\right\}, \quad y=\frac{1}{2}\left\{3 d_{j}-\sqrt{4 t_{j}-3 d_{j}^{2}}\right\}$.

Numerous devices for shortening the computations are suggested by numerical work, whether or not the prime factor resolution of $n$ be feasible.

As an immediate consequence of (2) we note that 9 is the only prime multiple of 9 which is the sum of two cubes $>0$; from (3) the only solution $x>0, y>0$ of $x^{3}+y^{3}=p^{2}, p$ prime, is $(x, y, p)=(1,2,3)$, etc. It is not difficult to obtain from (1) - (3) the known types of impossible equations $x^{3} \pm y^{3}=n$, except when $n$ is a cube, and some others that do not seem to have been stated.

The University of Washington

\title{
CONTACT CURVES OF THE RATIONAL PLANE CUBIC*
}

BY L. W. GRIFFITHS

1. Introduction. Contact conics and hyperosculating curves of the rational cubic have been discussed by Winger. $t$ Likewise some account has been given of curves of order $n$ which cut the cubic, rational or elliptic, in $(3 n-1)$ coincident points. $\$$ There remains the question of contact curves of order $n(n>2)$ whose contacts are of lower orders. This paper considers that question for the rational cubic, with results which hold for $n \geqq 1$ and for contacts of any order.

If the cubic is taken in the canonical form

$$
x_{1}=3 t^{2}, \quad x_{2}=3 t, \quad x_{3}=t^{3}+1,
$$

a necessary and sufficient condition that a set of $3 n$

* Presented to the Society, San Francisco Section, December 22, 1923.

$\dagger$ Involutions on the rational cubic, this Bulletin, vol.25 (1918), p. 27.

$\ddagger$ Winger, Some generalizations of the satellite theory, this Bulletin, vol. 26 (1919), p. 75. 
points on the cubic be the intersection of a curve* of order $n$ and the cubic is

$$
s_{3 n}=(-1)^{n},
$$

where $s_{3 n}$ is the product of the $3 n$ parameters of the points. The intersecting curve will be a contact curve if the $3 n$ points are not all distinct, i. e., if the exponent of at least one parameter in $s_{3 n}$ is $>1$. For instance, hyperosculating curves correspond to the coincidence case $t_{1}=t_{2}=\cdots=t_{3 n}$. It is from (2) that the results in this paper are derived.

2. An Example. As an example, consider a contact quartic and require that the 12 points common to it and the cubic coincide in two contacts, a 5-point contact at a point whose parameter is $\tau$ and a 7 -point contact at a point whose parameter is $t$. Then, in virtue of (2), $t$ and $t$ satisfy the equation

$$
x^{5} t^{7}=(-1)^{12} .
$$

Thus for a given $\boldsymbol{t}$ there are in fact seven points $t$, which means that seven quartics can have 5-point contact at an arbitrary point of the cubic and 7-point contact elsewhere. However, it is easily seen from (3) that $\boldsymbol{c}$ is merely one of a set of 5 points each of which determines this same set of 7 points $t_{i}$. That is, here are 35 related contact quartics, 5 with 7 -point contact at each $t_{i}$ and 7 with 5 -point contact at each $\tau_{i}$. It is easily verified also that the 12 parameters of the 12 points $\boldsymbol{r}_{i}$ and $t_{i}$ (in terms of $x$ ) satisfy (2). That is, the 12 contacts of the 35 related contact quartics lie on a non-contact quartic. But $\tau$ was an arbitrary point of the cubic. Therefore all contact quartics of the type illustrated are related in sets of 35 each, and the 12 contacts for any one set lie on a quartic.

Similar relations hold for other types of contact quartics. For instance, if $\boldsymbol{r}$ be supposed a 4-point contact and $t$ an 8-point contact, it can be shown that all contact quartics

* The curve will in fact be one of a pencil when $n>2$. Hereafter the word curve is used with this understanding. 
of this type are related in sets of 32 each, etc. Indeed, similar relations hold if and only if the 12 points common to the quartic and the cubic coincide in two contacts.

3. Generalization. These relations on contact quartics may be generalized immediately, for the method of proof is independent of the order $n$ of the intersecting curve. Here it is convenient to give the name complementary contacts to a pair of points like $\boldsymbol{r}$ and $t$ which absorb all the intersections of the curve and the cubic; and to indicate that a definite number $r<3 n$ of single intersections coincide at $\tau$ by saying that $\tau$ is a $P^{r}$ for the curve in question. Then two complementary contact curves are said to be of the same type if their values of $n$ and $r$ are respectively equal.

Theorem I. All complementary contact curves of the same type are related in sets of $r(3 n-r)$ each, where $r$ and $n$ have the values for the type in question. The distinct contacts for any one such set of complementary contact curves are $3 n$ in number, $r$ of them being contacts $P^{r}$ and the remaining $(3 n-r)$ being contacts $P^{3 n-r}$. Each point of the type $P^{r}$ is a contact $P^{r}$ for $(3 n-r)$ of the curves of the set and for no other complementary contact curves of this type in this set or any other; similarly each point of the type $P^{3 n-r}$ is a contact $P^{3 n-r}$ for $r$ of the curves of the set. That is, points of the cubic are related in nonoverlapping sets of $3 n$ each by complementary contact curves of a given type.

Theorem II. The $3 n$ contacts of a set of related complementary contact curves lie on a non-contact curve of order $n$ when and only when $n$ is even.

4. Several Sets of Contacts. In contrast to this relation (Theorem II) among the $3 n$ contacts of a single set of related complementary contact curves, there are relations between several such sets of contacts: for instance, in the satellite theory. In particular, the theorem that the tangentials of 3 points of a line are collinear relates 3 sets of contacts: each set consists of 3 points, a $P^{1}$ (the single 
intersection of a tangent and the cubic) and two $P^{2}$ 's (the contacts of tangents to the cubic from the $P^{1}$ ). It will be clear from Theorem 3 that the complete relation between these 3 sets of contacts is rather this theorem on tangentials and the less familiar theorem that the 6 contacts of tangents drawn from 3 collinear points lie by threes on 4 lines.

For the general case the several sets of contacts are determined as follows. First choose arbitrary integer values for $n, r<3 n, k$. Let $\boldsymbol{\tau}_{i}(i=1,2,3, \cdots, 3 k)$ be the $3 k$ distinct points in which a non-contact curve of order $k$ intersects the cubic. Then (Theorem I) determine the $3 n$ distinct contacts of the set of related complementary contact curves of order $n$ in which $\tau_{1}$ is a $P^{r}$; similarly determine the set in which $\tau_{2}$ is a $P^{r}$, etc. Since the $\boldsymbol{\tau}_{i}$ are distinct, there are $3 k$ distinct sets of complementary contact curves of the same type, each set of complementary contact curves having its set of $3 n$ contacts. These are the contacts whose relations are required. Note that they are $3^{2} k n$ distinct points, of which $3 k r$ are contacts of the type $P^{r}$ and $3 k(3 n-r)$ are contacts of the type $P^{3 n-r}$.

Theorem III. If, as above, any $3 k$ contacts of the type $P^{r}$ lie on a curve of order $k$, then the totality of contacts of the type $P^{r}$ lie on precisely $r^{3 k-1}$ curves of order $k$; and, further, the totality of contacts of the type $P^{3 n-r}$ lie on precisely $(3 n-r)^{3 k-1}$ curves of order $k$.

The proof consists in exhibiting two auxiliary sets of points, called the points $T_{i}$ and $T_{i}^{\prime}(i=1,2, \cdots, 3 k)$, such that (1) a necessary condition that the points $\boldsymbol{\tau}_{i}$ lie on a curve of order $k$ is that the points $T_{i}$ lie on a curve of order $k$; (2) a sufficient condition that another selection of points of the type $P^{r}$ lie on a curve of order $k$ is that the $T_{i}$ lie on a curve of order $k$; (3) a sufficient condition that the points $T_{i}^{\prime}$ lie on a curve of order $k$ is that the points $T_{i}$ lie on a curve of order $k$; and (4) a sufficient condition that a selection of $3 k$ points of the type $P^{3 n-r}$ lie on a curve of order $k$ is that the points $T_{i}^{\prime}$ lie on a curve of order $k$. Define $T_{i}=-\left(-\tau_{i}\right)^{r}$. Then if $S_{3 k}$ re- 
presents the product of the parameters of the points $T_{i}$

$$
S_{3 k}=(-1)^{k}(-1)^{k r}\left(\tau_{1} \tau_{2} \cdots \tau_{3 k}\right)^{r} .
$$

And so $S_{3 k}=(-1)^{k}$ if $\left(\tau_{1} \tau_{2} \cdots \tau_{3 k}\right)=(-1)^{k}$. This proves (1). The proof of (2) is more difficult, and requires the specific parameters of the $3 n$ contacts determined by $\tau_{i}$. By a method similar to that used in discussing quartics they are found to be

(5) $\left[\tau_{i}, \omega_{r} \tau_{i}, \omega_{r}^{2} \tau_{i}, \cdots, \omega_{r}^{r-1} \tau_{i}, t_{i}, \omega_{3 n-r} t_{i}, \omega_{3 n-r}^{2} t_{i}, \cdots, \omega_{3 n-r}^{3 n-r-1} t_{i}\right]$,

where $\omega_{r}$ is a primitive $r$ th root of unity, $\omega_{3 n-r}$ a primitive $(3 n-r)$ th root of unity, and $t_{i}=\left[(-1)^{n} / \boldsymbol{r}_{i}^{r}\right]^{1 /(3 n-r)}$, also a primitive root. Now select $(3 k-1)$ points, one from among $\left(\boldsymbol{\tau}_{1}, \omega_{r} \boldsymbol{\tau}_{1}, \cdots, \omega_{r}^{r-1} \boldsymbol{\tau}_{1}\right)$, one from among $\left(\boldsymbol{\tau}_{2}, \omega_{r} \boldsymbol{\tau}_{2}, \cdots, \omega_{r}^{r-1} \boldsymbol{\tau}_{2}\right)$, etc., omitting one from the set to which $\tau_{j}$ belongs. By these points a curve of order $k$ is determined. It will be shown that the $3 k$ th point $A$ in which this curve intersects the cubic is some one of the points $\left(\boldsymbol{\tau}_{j}, \omega_{r} \boldsymbol{\tau}_{j}, \cdots, \omega_{r}^{r-1} \boldsymbol{\tau}_{j}\right)$. For, determine the points $T$ for the points in which this curve intersects the cubic. They are $T_{1}, \ldots, T_{j-1}, T_{a}, T_{j+1}, \cdots, T_{3 k}$. But by the hypothesis and proof of (1) the $3 k$ points $T_{1}, \ldots, T_{j-1}, T_{j}, T_{j+1}, \ldots, T_{3 k}$ lie on a curve of order $k$. Since $(3 k-1)$ points on the cubic determine a curve of order $k, T_{a}$ is identical with $T_{j}$. Now from the definition of $T_{j}$ it is clear that each of the $r$ points $\left(\boldsymbol{\tau}_{j}, \omega_{r} \tau_{j}, \cdots, \omega_{r}^{r-1} \boldsymbol{\tau}_{j}\right)$ determines this same $T_{j}$, and that no other points determine this $T_{j}$. Therefore $A$ is some one of these points, say $\omega_{r}^{m} \tau_{j}$. But $A$ and the $(3 k-1)$ points selected lie on a curve of order $k$. Therefore (2) is proved. This method of proof of (2) leads to the enumeration $r^{3 k-1}$ stated in the theorem. For the choice from the points $\left(\boldsymbol{\tau}_{i}, \omega_{r} \boldsymbol{\tau}_{i}, \cdots, \omega_{r}^{r-1} \boldsymbol{\tau}_{i}\right)$ can be made independently in $r$ ways and independently of choosing from any of the other sets.

To prove (3) define $T_{i}^{\prime}=-\left(-t_{i}\right)^{3 n-r}$, where $t_{i}$ has the value in (5). Then each of the $(3 n-r)$ points $\left(t_{i}, \omega_{3 n-r} t_{i}, \cdots, \omega_{3 n-r}^{3 n-r-1} t_{i}\right)$ gives the same point $T_{i}^{\prime}$, and they are the only ones giving this $T_{i}^{\prime}$. Then if $S_{3 k}^{\prime}$ re- 
presents the product of the parameters of the points $T_{i}^{\prime}$, it can be shown that $S_{3 k}^{\prime}=1 / S_{3 k}$. Therefore (3) is proved. The proof of (4) is precisely the proof of (2), where $t_{i}$ and $T_{i}^{\prime}$ replace $\boldsymbol{r}_{i}$ and $T_{i}$ respectively. The enumeration $(3 n-r)^{3 k-1}$ is also immediate. The proof of Theorem 3 is therefore complete.

It should be noted that some of the results of Theorem 3, but no enumerations, follow from the theory of residuation.

5. Several Contacts of the Same Order. Finally, consider contact curves whose intersections are not completely absorbed in two contacts. Then at least two contacts are arbitrary, and relations are correspondingly indeterminate. The interesting case of precisely three contacts is that in which the contacts are of the same order; then from (2)

$$
\left(t_{1} t_{2} t_{3}\right)^{n}=(-1)^{n} \text {. }
$$

Thus the necessary and sufficient condition that a triad of points be contacts of this sort is that it belong to one of the $n$ involutions

$$
s_{3}=-\omega_{n}^{i}, \quad(i=0,1, \cdots, n-1),
$$

where $\omega_{n}$ is a primitive $n$th root of unity. For $n=2$ these contact curves are the familiar tritangent conics.

The interesting case of $n$ contacts is also that in which the contacts are of the same order, when from (2)

$$
\begin{gathered}
\left(t_{1} t_{2} \cdots t_{n}\right)^{3}=(-1)^{n}, \\
s_{n}=(-1)^{n} \omega_{3}^{i}, \quad(i=0,1,2),
\end{gathered}
$$

where $\omega_{3}$ is a primitive cube root of unity.

6. Analogous Theorems for the General Cubic. For the general cubic, expressed rationally in terms of $\wp(u)$, analogous theorems hold. The equations in terms of the elliptic argument are not of the simple type (2) afforded by the rational cubic, but yield determinate enumerations.

The University of Washington 